IISc-CHEP/06/07

LAPTH-1183/2007

\title{
Top production at the Tevatron/LHC and nonstandard, strongly interacting spin one particles
}

\author{
Debajyoti Choudhury $^{a}$, Rohini M. Godbole ${ }^{b, c}$, Ritesh K. Singh $^{d}$ and Kshitij Wagh ${ }^{e}$ \\ ${ }^{a}$ Dept. of Physics and Astrophysics, University of Delhi, Delhi 110 007, India. \\ ${ }^{b}$ Centre for High Energy Physics, Indian Institute of Science, Bangalore, 560 012, India. \\ ${ }^{c}$ Dept. of Theoretical Physics, Tata Institute of Fundamental Research, \\ Mumbai 400 005, India. \\ ${ }^{d}$ Laboratoire d'Annecy-Le-Vieux de Physique Theorique (LAPTH), Chemin de Bellevue, B.P. 110, \\ F-74941 Annecy-le-Vieux, Cedex, France. \\ ${ }^{e}$ Dept. of Physics and Astronomy, Rutgers University, Piscataway, \\ New Jersey 08854-8019, U.S.A.
}

\begin{abstract}
In this note, we consider possible constraints from $t \bar{t}$ production on the gauge bosons of theories with an extended strong interaction sector such as axigluons or flavour universal colorons. Such constraints are found to be competitive with those obtained from the dijet data. The current $t \bar{t}$ data from the Tevatron rule out axigluon masses $\left(m_{A}\right)$ up to $910 \mathrm{GeV}$ and $920 \mathrm{GeV}$ at $95 \%$ and $90 \%$ confidence levels respectively. For the case of the flavour universal colorons, for $\cot \xi=1$, where $\xi$ is the mixing angle, the mass ranges $m_{C} \lesssim 800 \mathrm{GeV}$ and $895 \lesssim m_{C} \lesssim 1960 \mathrm{GeV}$ are excluded at $95 \%$ confidence level (C.L.), whereas the same at $90 \%$ C.L. are $m_{C} \lesssim 805 \mathrm{GeV}$ and $880 \lesssim m_{C} \lesssim 2470$ $\mathrm{GeV}$. For $\cot \xi=2$ on the other hand, the excluded range is $m_{C} \lesssim 955(960) \mathrm{GeV}$ and $1030 \lesssim m_{C} \lesssim 3250\left(1020 \lesssim m_{C} \lesssim 3250\right) \mathrm{GeV}$ at $95 \%$ (90\%) C.L. respectively. We point out that for higher axigluon/coloron masses, even for the dijet channel, the limits on the coloron mass, for $\cot \xi=1$, may be different than those for the axigluon. We also compute the expected forward-backward asymmetry for the case of the axigluons which would allow it to be discriminated against the SM as also the colorons. We further find that at the LHC, the signal should be visible in the $t \bar{t}$ invariant mass spectrum for a wide range of axigluon and coloron masses that are still allowed. We point out how top polarisation may be used to further discriminate the axigluon and coloron case from the SM as well as from each other.
\end{abstract}




\section{Introduction}

The Standard Model (SM) has had unprecedented success in passing precision tests at the SLC, LEP, HERA and the Tevatron. The agreement between the directly measured value of the top mass, $m_{t}$, and the one indicated by precision measurements [1, 2], has played a crucial role in this test of the SM to the loop level, providing indirect evidence for the Higgs boson. However, a direct verification of the Higgs mechanism of the spontaneous breaking of the Electroweak (EW) symmetry, thereby providing an understanding of the mechanism of the mass generation for fermions, is still lacking. Its understanding will be one of the focal points of the investigations at the Large Hadron Collider (LHC) and, thereafter, the International Linear Collider(ILC) [3]. The top quark, with a mass very close to the electroweak symmetry breaking scale, is expected to provide a probe for understanding the phenomenon of symmetry breaking in the SM. For the same reason, any alternative to the Higgs mechanism almost always involves the top quark [4. Thus, a study of production and properties of the top quark at the $\mathrm{TeV}$ colliders can be used as a 'low' energy probe for any ('high scale') new physics beyond the SM, as well as a good probe of alternates to the Higgs mechanism. An accurate testing of the mass relations between $m_{t}$ and $m_{W}$, as predicted in the SM, is an important part of the physics program of any $\mathrm{TeV}$ energy collider [5]. Clearly, top physics is a very promising place to look for new physics effects [6]. Already at the Tevatron, this has been a very fruitful area of investigations [7, 8] and one expects a top factory such as the LHC to be a goldmine for studying the $\mathrm{SM}$ as well as the beyond the $\mathrm{SM}(\mathrm{BSM})$ physics [9]. Recent discussions in the literature have addressed the possibility of using $t \bar{t}$ production at the Tevatron for putting 'direct' constraints [10] on the Kaluza Klein(KK) gluons of the bulk Randall-Sundrum Model, using their large coupling to the $t \bar{t}$ pairs to probe the same at the LHC through use of $t$ polarisation, in $t \bar{t}$ production [11, 12], as well as using spin-spin correlations in $t \bar{t}$ production via KK excitations of the graviton [13]. $t \bar{t}$ pair production at the Tevatron through a $Z_{t}^{\prime}$ in various versions of Topcolor models has also been discussed [14]. The feasibility of using the $t$ polarisation in $t \bar{t}$ production via an EW resonance such as an additional $Z^{\prime}$ occurring in (say) unified models or a $Z_{H}$ which occurs in the Little Higgs models has also been recently explored [15]. In this note, we revisit the issue of constraints that the current measurements of $t \bar{t}$ production at the Tevatron [7, 8] imply for theories which have a strongly interacting spin-1 particle in addition to the gluons. We discuss the $t \bar{t}$ production for the cases of massive coloured 'axigluons' which exist in theories with chiral colour [16, 17] and that of the flavour universal colorons [18] which exist in certain versions of extended colour gauge theories. It may be mentioned here that the case of axigluons and the flavour universal colorons differs from the other strongly interacting bosons such as the KK gluons or the ETC theories, in that they do not have preferential coupling to $t \bar{t}$. Thus, the sensitivity of $t \bar{t}$ production to our case should be expected to differ from that for such models with enhanced top-couplings.

Below, we first summarise the relevant details of the two models as well as the current limits on

the masses of the axigluons and flavour universal colorons. Then we will show the constraints the 
current data on $t \bar{t}$ production imply for these. We then look at the phenomenology of the colorons and the axigluons at the LHC as well.

\section{Models.}

In the unifiable chiral colour model [16, 17], the high energy strong interaction gauge group $S U(3)_{L} \times$ $S U(3)_{R}$ is spontaneously broken to the usual $S U(3)_{L+R}$ and thus one has an octet each of massless gluons $(g)$ and massive axigluons $(A)$ with an axial vector coupling, $\frac{1}{2} g_{s} \gamma_{\mu} \gamma_{5} \lambda^{a}$, where $g_{s}$ is the usual strong coupling and $\lambda_{a}$ the usual Gell-Mann matrices. The above mentioned axigluon is common to all the different versions of the chiral colour models that exist. The strong coupling of $A$ to $q \bar{q}$ ensures large production cross-sections for the axigluons at a hadronic collider, at the same time causing the axigluon to have a reasonably large width. Considerations of embedding the chiral colour group into a grand unified theory implied a 'natural' value for the axigluon mass of the order of the weak scale $\sim 250$ $\mathrm{GeV}$. It was soon realised that not only could the axigluon be searched for in the dijet channel [19] through the process $p \bar{p}(p) \rightarrow A^{*} \rightarrow q \bar{q}$, but also that the forward-backward asymmetry caused by the interference between the $g$ and $A$ contributions to $q \bar{q} \rightarrow Q \bar{Q}$ [20] could be used to probe and constrain the $A$ contribution. The Tevatron searches for new resonances decaying to dijets $[21,22$, exclude the mass range $200<m_{A}<1130 \mathrm{GeV}$. The lighter axigluon windows were eliminated by various considerations such as hadronic decays of the $Z$-boson etc. [23, 24]. $t \bar{t}$ production through the process $q \bar{q} \rightarrow t \bar{t}$ can be used very effectively for the axigluon search due to large $q \bar{q}$ fluxes at the Tevatron. The large top-sample at the Tevatron was shown capable of providing reach of the order of $1 \mathrm{TeV}$ for generic new physics [6]. The early Tevatron data on top-pair production was shown [25] to disfavour the contribution from a lighter axigluon mass window $50<m_{A}<120 \mathrm{GeV}$ (which was then not completely ruled out) at about $1.5 \sigma$ level. The analysis can be further sharpened by using the forward-backward asymmetry in the $t \bar{t}$ production.

The flavour universal coloron model [18] belongs to a class of models of extended colour which arose from the general effort to understand the mechanism of EW symmetry breaking and the large mass of the top, $m_{t}$, in the same framework, and wherein a top quark condensate enhances the top quark mass and drives the EW symmetry breaking. Specific examples of this idea are topcolor [26, 6] and topcolor assisted technicolor [27]. In general, in the coloron models, it is assumed that the colour group at high energies is larger, given by $S U(3)_{I} \times S U(3)_{I I}$, and breaks at the TeV scale to the usual $S U(3)_{c}$, giving rise to an octet of massive, strongly interacting gauge bosons, called colorons. Variants of the coloron models differ in how the different generations of quarks couple to $S U(3)_{I}$ and $S U(3)_{I I}$. In the original model [26, 6], the first two families couple to $S U(3)_{I}$ and the third couples to $S U(3)_{I I}$. The phenomenology of such a coloron at the Tevatron and at the LHC, with respect to the $t \bar{t}$ final states has been discussed [6, 28]. The Universal flavour coloron model [18, 29], is a variant of this idea, where all the quarks transform as a $(1,3)$ under this extended gauge group and the two gauge couplings are $\xi_{1}, \xi_{2}$ for $S U(3)_{I}$ and $S U(3)_{I I}$ respectively, with $\xi_{1} \ll \xi_{2}$. The massive colorons couple 
to all the quarks through a $\frac{1}{2} g_{s} \cot \xi \gamma_{\mu} \lambda^{a}$ coupling, where $\xi$ is the mixing angle given by $\cot \xi=\xi_{2} / \xi_{1}$. The mass of the coloron $m_{C}$ is related to $\xi$, the strong coupling $g_{s}$ and the vacuum expectation value of the scalar $\Phi$ which transforms as a $(3, \overline{3})$ under the extended gauge group and breaks it down to $S U(3)_{c}$. The coloron, like the axigluon, is also a broad resonance. The model with flavour universal coloron can be grafted rather nicely onto the standard one-Higgs-doublet model of EW physics and has a naturally heavy top quark [30]. Electroweak precision measurements constrain the $\rho$ parameter and hence the model parameter space, the constraint being given by $M_{c} / \cot \xi \gtrsim 450 \mathrm{GeV}[31] 1$. Further, the value of $\cot \xi$ is limited from above to $\sim 4$, by the requirement that the model remains in the Higgs phase.

The colorons will contribute to the dijet production [29, 32] in almost the same way as the axigluons. The constraints on $m_{A}$ from the dijet data [21, 22] can also be translated into constraints on $m_{C}$. However, the coloron contribution will not give rise to any forward-backward asymmetry in $q \bar{q}$ production as opposed to the case of the axigluons. Ref. [29] estimated the possible constraints that may be obtained with the $b$-tagged dijets, using the analysis constraining the topgluon production and decay, accounting for the difference in the possible decay channels and the width in the two cases. Further, it was claimed [29] that while using the approximation of incoherent sum of the background and the signal, the signal strengths in the dijet channel for the flavour universal coloron for $\cot \xi=1$ will be the same as that of an axigluon of the same mass. It was further claimed [29], that since the expected cross-sections for the coloron, in the above approximation, increase with cot $\xi$, the constraints implied by the dijet analysis for the axigluons also give the most conservative constraint for the coloron. At present, the best quoted bounds for axigluon and colorons come from the Tevatron dijet data which rule out masses up to $\simeq 980 \mathrm{GeV}[22$, 24]. As we point out later, for heavier coloron masses, some of the above statements need to be amended.

\section{Axigluon and Coloron contribution to $t \bar{t}$ production at hadronic colliders.}

At the tree level, the presence of an axigluon $A$ or the coloron $C$ can affect $t \bar{t}$ production only as far as the $q \bar{q}$-initiated subprocess is concerned, leaving the $g g$-initiated subprocess unaltered. Since the $q \bar{q}$ fluxes are dominant over the $g g$ fluxes at the Tevatron, the possible presence of a axigluon/coloron resonance (the $s$-channel $q \bar{q} \rightarrow A^{*}\left(C^{*}\right) \rightarrow t \bar{t}$ diagram is the only new contribution) can affect the total rate of $t \bar{t}$ production significantly. This can be easily understood by realising that, at the Tevatron, even in the absence of these exotic bosons (i.e., even for the SM alone), the contribution to the total $t \bar{t}$ cross-section from the $q \bar{q}$ initial state dominates over the $g g$ contribution by a factor of $\sim \mathcal{O}(10)$, depending on the PDF's, choice of scale etc.

In the presence of an axigluon, the parton-level differential cross section for the $q \bar{q}$-initiated process

\footnotetext{
${ }^{1}$ We have checked that current precision measurements also imply a similar constraint.
} 
is modified and for final state $t(\bar{t})$ with helicity $\lambda(\bar{\lambda})$ is given by,

$$
\begin{aligned}
\frac{d \sigma}{d \hat{t}}(q \bar{q} \rightarrow t(\lambda) \bar{t}(\bar{\lambda}))=\frac{\pi \alpha_{s}^{2}}{9 \hat{s}^{2}}\{ & {\left[(1+\lambda \bar{\lambda}) \frac{4 m_{t}^{2}}{\hat{s}}\left(1-c_{\theta}^{2}\right)+(1-\lambda \bar{\lambda})\left(1+c_{\theta}^{2}\right)\right] } \\
& \left.+\frac{(1-\lambda \bar{\lambda})}{\left(\hat{s}-m_{A}^{2}\right)^{2}+\Gamma_{A}^{2} m_{A}^{2}}\left[\hat{s}^{2} \beta^{2}\left(1+c_{\theta}^{2}\right)+4 \hat{s}\left(\hat{s}-m_{A}^{2}\right) \beta c_{\theta}\right]\right\},
\end{aligned}
$$

where $\hat{s}$ is the invariant mass of the $t \bar{t}$ system with $\beta\left(\equiv \sqrt{1-4 m_{t}^{2} / \hat{s}}\right)$ and $\theta$ being the top-velocity and the scattering angle in the parton centre of mass frame respectively; $\lambda$ and $\bar{\lambda}$ which are the helicities (as distinct from chirality) of the top and the anti-top, and take values \pm 1 . Note that there are no terms linear in the helicities, as would have been present if the intermediate boson (axigluon) were to have both vectorial and axial couplings. The width $\Gamma_{A}$ is given by

$$
\Gamma_{A} \equiv \sum_{q} \Gamma(A \rightarrow q \bar{q}) \approx \frac{\alpha_{s} m_{A}}{6}\left[5+\left(1-\frac{4 m_{t}^{2}}{m_{A}^{2}}\right)^{3 / 2}\right] \text {. }
$$

Summing over the top polarizations, the expression for the cross-section for $t \bar{t}$ production for the $q \bar{q}$ initial state becomes,

$$
\begin{aligned}
\frac{d \sigma}{d \hat{t}}(q \bar{q} \rightarrow t \bar{t})=\frac{2 \pi \alpha_{s}^{2}}{9 \hat{s}^{2}} & \left\{\left[\frac{4 m_{t}^{2}}{\hat{s}}\left(1-c_{\theta}^{2}\right)+\left(1+c_{\theta}^{2}\right)\right]\right. \\
+ & \left.\frac{\hat{s}^{2} \beta^{2}\left(1+c_{\theta}^{2}\right)+4 \hat{s}\left(\hat{s}-m_{A}^{2}\right) \beta c_{\theta}}{\left(\hat{s}-m_{A}^{2}\right)^{2}+\Gamma_{A}^{2} m_{A}^{2}}\right\},
\end{aligned}
$$

and reduces to Eq. [2.2] of [19] in the limit of zero quark (top) mass. Note the existence of the term odd in $c_{\theta}$ here, which is due to the interference between the gluon and the axigluon amplitude. We may mention here that our Eq. 3 does not agree with Eq.[2] of Ref. [20]. The interference term in their Eq. [2] is proportional to $\beta^{2}$ instead of $\beta$ as in our Eq. 3. further as per their Eq.[2] the square of the axigluon exchange amplitude is proportional to the gluon exchange amplitude, apart from the obviously different propagator, which can not be true for massive quarks in the final state. Needless to mention of course that the first term in eq. 3, corresponding to the SM, agrees with the standard QCD expression [33, now available in textbooks on the subject. Since the contribution of the $g g$ initial state [33] (to be added incoherently) remains unchanged we refrain from reproducing the formulae here.

In the presence of the coloron, the differential cross-section for production of production of $t(\bar{t})$ with helicities $\lambda(\bar{\lambda})$ respectively, reads for the $q \bar{q}$ initial state:

$$
\begin{aligned}
& \frac{d \sigma}{d \hat{t}}(q \bar{q} \rightarrow t(\lambda) \bar{t}(\bar{\lambda}))=\frac{\pi \alpha_{s}^{2}}{9 \hat{s}^{2}}\left\{(1+\lambda \bar{\lambda}) \frac{4 m_{t}^{2}}{\hat{s}}\left(1-c_{\theta}^{2}\right)+(1-\lambda \bar{\lambda})\left(1+c_{\theta}^{2}\right)\right\} \\
& \left|1+\frac{\hat{s} \cot ^{2} \xi}{\hat{s}-m_{C}^{2}+i \Gamma_{C} m_{C}}\right|^{2} .
\end{aligned}
$$


The width is now given by

$$
\Gamma_{C} \equiv \sum_{q} \Gamma(C \rightarrow q \bar{q})=\frac{\alpha_{s} \cot ^{2} \xi}{6} m_{C}\left\{5+\left[1+2 \frac{m_{t}^{2}}{m_{C}^{2}}\right]\left(1-\frac{4 m_{t}^{2}}{m_{C}^{2}}\right)^{1 / 2}\right\} .
$$

Once again, summing over the polarization gives us for the differential cross-section

$$
\frac{d \sigma}{d \hat{t}}(q \bar{q} \rightarrow t \bar{t})=\frac{2 \pi \alpha_{s}^{2}}{9 \hat{s}^{2}}\left\{\frac{4 m_{t}^{2}}{\hat{s}}\left(1-c_{\theta}^{2}\right)+\left(1+c_{\theta}^{2}\right)\right\}\left|1+\frac{\hat{s} \cot ^{2} \xi}{\hat{s}-m_{C}^{2}+i \Gamma_{C} m_{C}}\right|^{2} .
$$

As far as the coloron is concerned, the net effect of the addition of a simple vectorial interaction is to just change the propagator from $1 / \hat{s}$ to $1 / \hat{s}+\cot ^{2} \xi /\left(\hat{s}-m_{C}^{2}+i m_{C} \Gamma_{C}\right)$ [ 6 , in the $q \bar{q} \rightarrow t \bar{t}$ amplitude. Our Eq. 6] agrees with Eq.[3.7] of Ref. [29] and also with Eq.[3.3], the corresponding expression for dijet cross-section, when the limit of zero top mass is taken. In the absence of the coloron contribution the term in Eq. 6 proportional to $\cot \xi$ is absent and then the expression trivially reduces to the usual QCD contribution for the $q \bar{q}$ initial state [33]. Again the presence of colorons does not modify the contribution of the $g g$ initial state from its SM form.

We now comment on certain differences between the two cases (axigluons vs. colorons) as also on the (non-)applicability of certain approximations made in the literature in the context of dijet production. For one, a comparison of Eqs.( 2 \& 5), shows that the two decay widths differ for $m_{A}\left(m_{C}\right) \gtrsim 2 m_{t}$ (a range not the focus of discussions in Refs. [19, 29]) even for $\cot \xi=1$. This becomes even more pronounced when we consider the branching fraction into a $t \bar{t}$ pair (see Fig $1 a$ ). Thus the branching fraction into dijets will also be different for a coloron with $\cot \xi=1$ from an axigluon of the same mass, for boson masses $\gtrsim 2 m_{t}$, particularly in the range $0.5 \mathrm{TeV}<m_{\text {boson }}<1.5$ $\mathrm{TeV}$, the relative difference in the branching fractions however being smaller than that for the $t \bar{t}$ case. Furthermore, the large widths immediately imply that the (narrow-width) approximation of resonant production and subsequent decay is no longer a good one. This is borne out by Fig $1 b$, wherein we compare the $t \bar{t}$ cross-section at the Tevatron expected, for an axigluon, in the resonant (incoherent) approximation with the exact value of the deviation in $t \bar{t}$ cross section obtained by integrating over the (large) width of the resonance. To be more specific, we compare $\sigma(A) \times B R(A \rightarrow t \bar{t})$ with $\delta \sigma \equiv \sigma_{A}(t \bar{t})-\sigma_{S M}(t \bar{t})$. One sees that the approximation could underestimate the cross-section by up to a factor of 3-4, even for axigluon masses as low as $\sim 1 \mathrm{TeV}$, which are well within the reach of the Tevatron. For the case of the coloron, the situation is even more complicated as we discuss below.

The differential cross-sections for the two cases differ markedly in the interference term between the gluon-exchange and the nonstandard strong gauge boson (axigluon or coloron)-exchange amplitudes. For the axigluon, this contribution, being a parity-odd one, does not contribute to the total cross section, but gives rise to a forward-backward asymmetry. For the coloron, on the other hand, the interference term does indeed contribute to the total cross section and thus is of great importance when integrating over the non-resonant contributions. Note further that the interference terms between the 

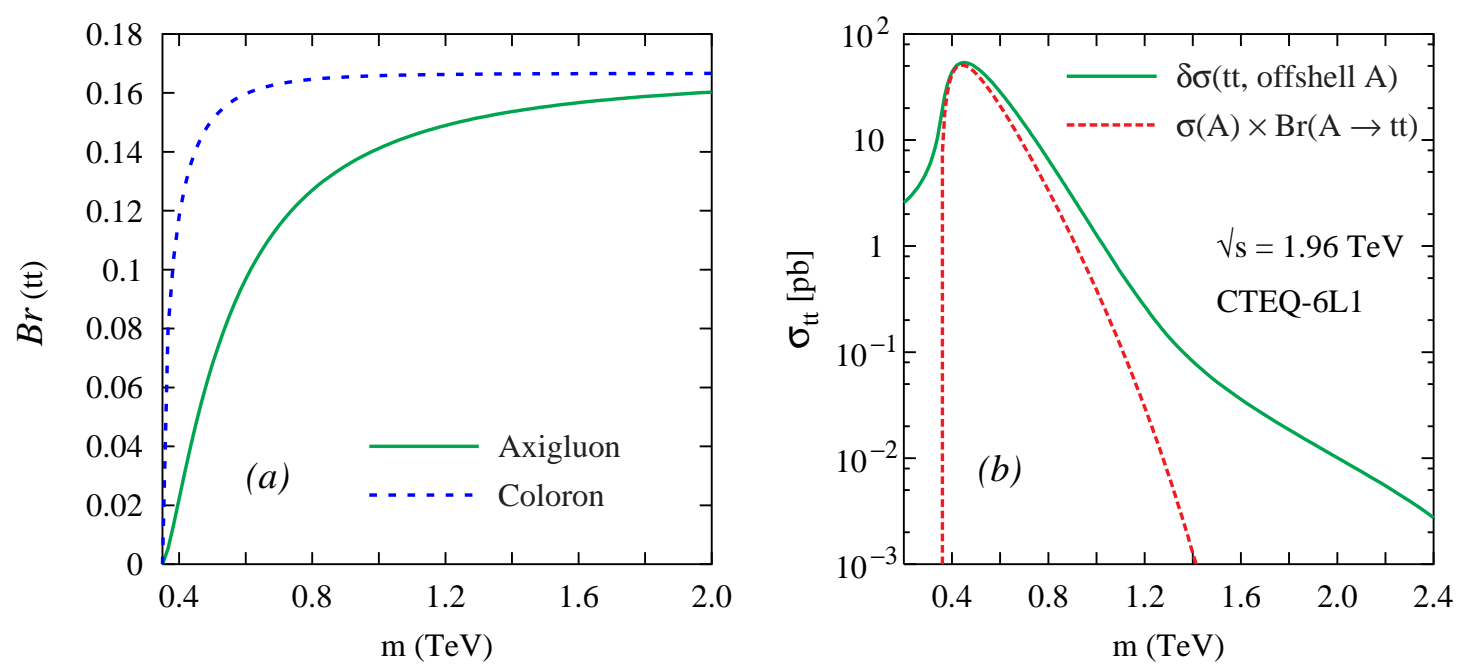

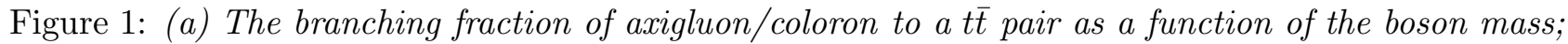
(b) A comparison of the deviation of the total $t \bar{t}$ cross section caused by the presence of an axigluon (solid line) with the resonant production followed by decay.

coloron and gluon-exchange contributions changes sign as the subprocess centre of mass energy $\sqrt{\hat{s}}$ passes through $m_{C}$, and, depending on the value of $\cot \xi$, can even reduce the total integrated crosssection below the SM value. Thus, the interference term has different behaviour for the coloron and the axigluon. As can be also seen from Eqs. 3 and 6, even the term in the cross-section proportional to the square of the propagator of the unstable strong boson (axigluon or coloron) is different in the two cases for massive quarks in the final state.

The observations above have several implications. First, it is clear that for $m_{A}, m_{C} \lesssim 1.5 \mathrm{TeV}$, as far as the $t \bar{t}$ production cross-section is concerned, (even on resonance) the expectations for a coloron with $\cot \xi=1$ will be different from that for an axigluon of the same mass, unlike the case of dijet cross-sections where only massless quarks are involved. Even for the latter case, for the heavier colorons now being looked for in the Tevatron dijet data, for $\cot \xi=1$, the $\sigma \times B$, is no longer the same as that of an axigluon of the same mass. As was already mentioned, an equality between these, claimed in Ref. [29] and used in all the analyses [22, 24] so far, is true only for relatively low values of the boson masses. It may be pointed that even in that case the (approximate) equality is true only when the resonant contribution dominates and one considers only the incoherent sum of background and the signal. With increasing mass of the boson, the width increases, necessitating the inclusion of the interference term to the total cross-section and the approximation of incoherent sum of the background and the signal is no longer valid. For the case of the colorons, the effect of the parity-even interference term gives a non monotonic dependence of the total integrated cross-section on $m_{C}$ and $\cot \xi$. Thus it means that for heavier colorons, a value of mass $m_{C}$ excluded at $\cot \xi=1$ need not be 
excluded at higher values of $\cot \xi$.

\section{Numerical Results and constraints from $t \bar{t}$ production at Tevatron}

Using the formulae presented in section 3 (and including the contribution from the $g g$ initial state [33]), we now proceed to calculate the $t \bar{t}$ cross-section at the Tevatron Run II $(\sqrt{s}=1.96)$ and assess the implications of the current data. For all our computations, we use the CTEQ-6L1 parton distributions [34], with a choice of $Q^{2}=m_{t}^{2}$ for the factorization scale and $m_{t}=175 \mathrm{GeV}$. For the SM process, corresponding $K$-factor corresponding to this choice of PDF and the scale, amounts to 1.08 [35]. As mentioned earlier, at the Tevatron, the SM production process is dominated by the $q \bar{q}$ initial states, and since both the color and tensorial structure of the process under consideration is very similar to the SM process, it is expected that the use of the SM $K$-factor for the cross-section including the effects of the axigluon/coloron contribution is well justified. This is what we shall use henceforth. We have verified that except very close to the peaks, the cross-sections are stable with respect to a change in the scale of the hard process (and/or the choice of the parton distributions) as long as the corresponding correct $K$-factor [35] is used. While the smallness of $K(1.08)$ for our choice of parton density, is a reflection of the rather large value of $\alpha_{s}\left(M_{Z}\right)=0.130$ used in CTEQ-6L1, note that this choice of parton densities is not a special one.

In Fig 2, the solid (green) line shows our predictions for the $t \bar{t}$ production cross-section as a function of the axigluon mass. This may be compared with the current experimental data which gives (CDF Run II results averaged over all channels) [7]

$$
\sigma(p+\bar{p} \rightarrow t+\bar{t}+X ; \sqrt{s}=1.96 \mathrm{TeV})=7.3 \pm 0.5(\text { stat }) \pm 0.6(\text { syst }) \pm 0.4(\text { lum }) \mathrm{pb} .
$$

In Fig, 2, the central value is denoted by the horizontal grey dashed line, whereas the sidebands (dashed black and magenta lines respectively) give the 95\% -Confidence Level (C.L.) limits (obtained by adding the errors in quadrature). As can be seen from the plot, the data rule out axigluon masses $\left(m_{A}\right)$ up to $910 \mathrm{GeV}$ at $95 \%$ C.L. It can be easily seen that these are comparable to the current constraints [22, 24] available from the Tevatron dijet data. As already mentioned before, predictions for the $t \bar{t}$ production for the coloron (even for $\cot \xi=1$ ) differ from that for the axigluon of the same mass. In particular, due to the destructive (parity-even) interference for $\hat{s}<m_{C}^{2}$, the cross-section shows a dip as a function of $m_{C}$ and rises again. That the extent of this interference depends crucially on the magnitude of $\cot \xi$ can be understood from the discussion following Eq.(6). As can be clearly seen, in this case, the data rule out values of $m_{C}$ below $800 \mathrm{GeV}$ at $95 \%$ confidence level and also the mass range between 895 $\mathrm{GeV}$ to $1960 \mathrm{GeV}$ at $95 \%$ confidence level, for $\cot \xi=1$. At $90 \%$ C.L. the exclusion is for $m_{C}<805$ $\mathrm{GeV}$ and for masses between $880 \mathrm{GeV}$ to $2470 \mathrm{GeV}$. For $\cot \xi=2$ on the other hand, the excluded range at $95 \%$ C.L. is $m_{C} \lesssim 955 \mathrm{GeV}$ and $1030 \lesssim m_{C} \lesssim 3250 \mathrm{GeV}$. At $99.99 \%$ C.L., the same is, e.g., $m_{C} \lesssim 930 \mathrm{GeV}$ and $1110 \lesssim m_{C} \lesssim 1860 \mathrm{GeV}$. 


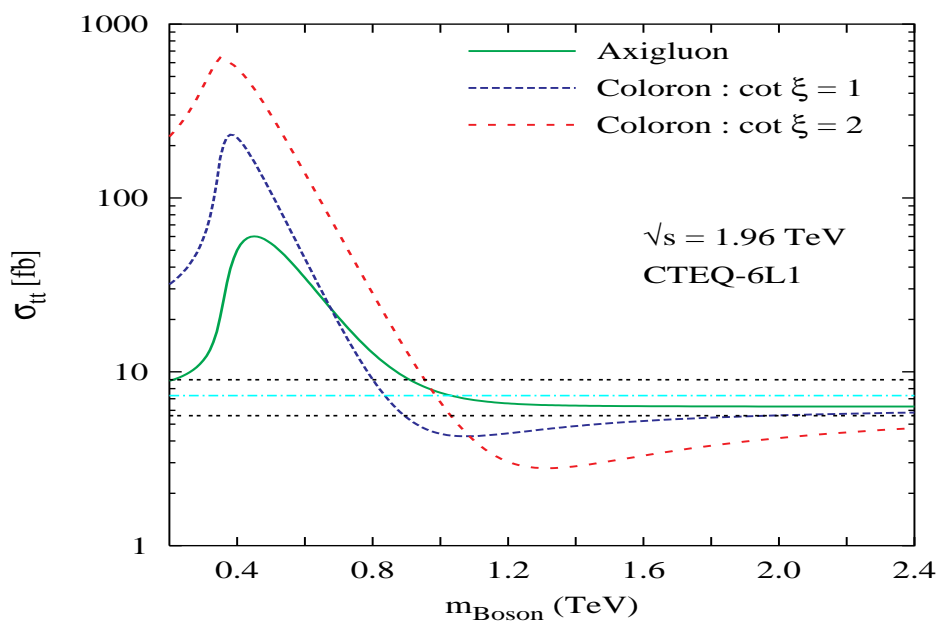

Figure 2: $t \bar{t}$ production cross-section at the Tevatron as a function of the axigluon (coloron mass) and constraints on it from the current $t \bar{t}$ production data. The solid (green) line corresponds to the axigluon case. The short- (blue) and long-dashed (red) lines correspond to the flavour universal coloron for $\cot \xi=1$ and $\cot \xi=2$ respectively. The horizontal lines correspond to the current central value from the CDF experiment [7] and the 95\% confidence level bands. We have used CTEQ-6L1 parton distribution functions evaluated at $Q=m_{t}$ and included the appropriate $K$-factor [35].

It should be noted that the fact of the consistency of certain domains of model parameters with the data cannot be interpreted as evidence for the existence of colorons, simply because the data are also consistent with the SM predictions as well. Rather, the Tevatron data may be used to rule out regions in the $m_{C}$-cot $\xi$ plane and we display the corresponding results in Fig.3. Once again, we are witness to the rôle of the aforementioned interference effects. The thin white sliver is consistent with the Tevatron data at the $1 \sigma$ level. Thus the values of $\cot \xi-m_{C}$ lying in this sliver are consistent with the current data. (Note that any quantitative statement depends rather critically on the exact value of $m_{t}$ used, due to the strong dependence of the cross-section on $m_{t}$ near the edge of the phase space boundary, as the case is at the Tevatron.) As one goes out of the region of the sliver, we find that the regions of the $\cot \xi-m_{C}$ plane are excluded as they predict cross-sections either above or below the upper and lower bound on the experimentally measured $t \bar{t}$ cross-section of Eq. 7 at a given level of confidence. Thus excluded regions on either side of the thin sliver, are excluded either from above (the cross-section being too high) or from below (the cross-section being too low). As one moves away to the left of this sliver, the cross section increases sharply, leading to the very thin bands as depicted in Fig 3. Moving to the right though, the cross section initially falls, and then rises again, both changes being slower 


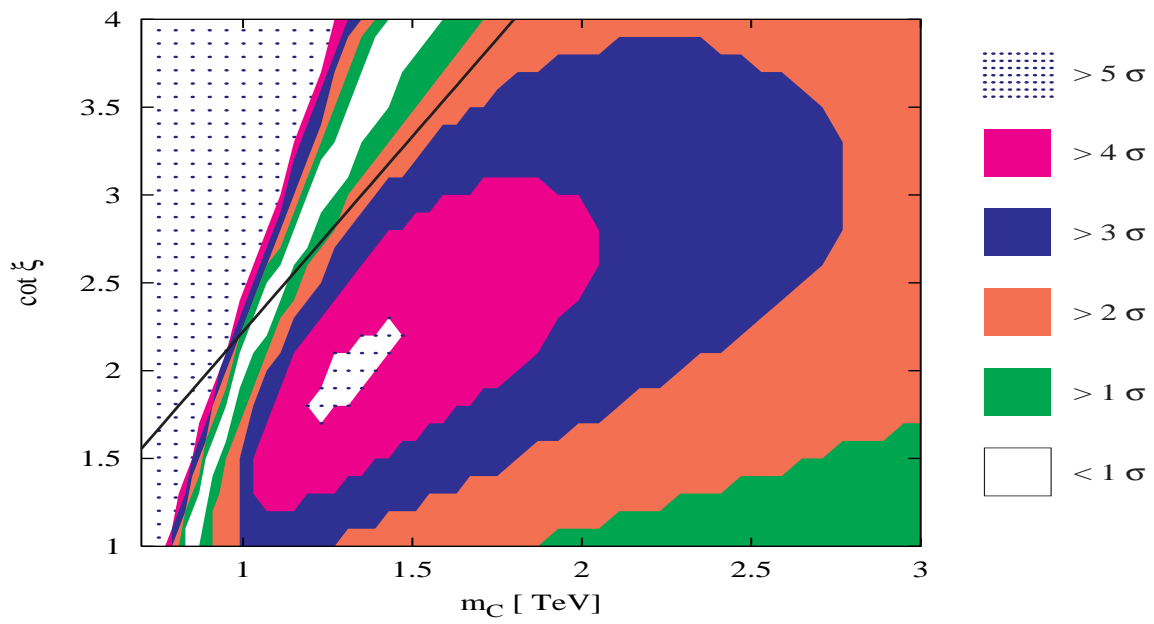

Figure 3: Exclusion region in the $\cot \xi-m_{C}$ plane using the $t \bar{t}$ data at Tevatron [7]. The solid curve shows the constraint imposed by the $\rho$ parameter $m_{C} / \cot \xi \gtrsim 450$. We restrict the plot to $\cot \xi<4.0$ for reasons mentioned in the text.

than that to the left of the $1 \sigma$ sliver. The different rate of change of the cross-section with $m_{C}$ to the left and right of the sliver is a reflection of the behaviour already seen in Fig 2, it can be traced back to the interplay of the interference effect - as in Eq.(6) - and the parton density distributions. More concretely, this results in the iso-cross section contours being closed curves roughly focused at $\sim(1.2$ $\mathrm{TeV}, 1.7)$. Indeed, the bands to the left of the $1 \sigma$ sliver, and the sliver itself, are but parts of larger closed bands, not quite apparent in the figure as we have restricted the range of $\cot \xi$ such that one always stays within the Higgs phase of the model [29]. The figure is also reflective of the fact that, for a given $m_{C}$, the cross-section could be a non-monotonic function of $\cot \xi$. For small $m_{C}$, the cross section does increase monotonically with $\cot \xi$, but for larger values of $m_{C}$, the cross-section first falls as we increase $\cot \xi$ from unity (a reflection of the destructive interference operative for $\hat{s}<m_{C}^{2}$ ) and then increases once the coloron amplitude overwhelms the gluon amplitude. Such effects, for example, result in the island of $5 \sigma$ exclusion approximately centred at $\sim(1.2 \mathrm{TeV}, 1.7)$. Also shown in Fig 3 is the limit imposed by the $\rho$ parameter, namely $m_{C} / \cot \xi \gtrsim 450 \mathrm{GeV}$; we see that even below this line there is a patch of white ( $1 \sigma$ agreement).

Apart from the above mentioned differences between the axigluon and coloron in the total crosssection, the two cases are also distinguished by the parity odd and even nature of the interference term, as was already mentioned. This gives rise to a forward-backward (FB) asymmetry [20] at the Tevatron for the production of dijets as well as heavy quarks in the final state. For the latter, the FB 
asymmetry can be measured using the decay leptons from the heavy quark, in the present case the $t$ quark. The numerical value for this asymmetry had been calculated in Ref. [25] using the formulae of Ref. [20], which are incorrect. We present, in Fig, 4, the FB asymmetry expected at the Tevatron,

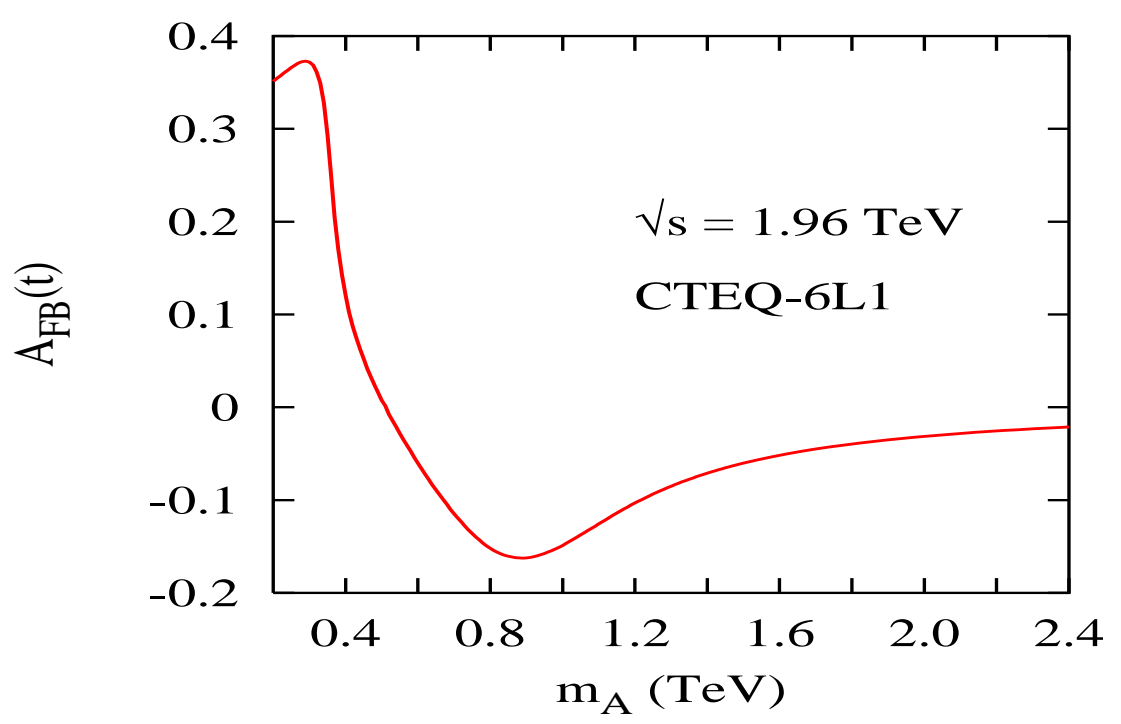

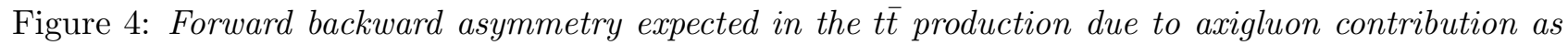
a function of the axigluon mass $m_{A}$.

calculated using our Eq. 3, as a function of $m_{A}$. Note again that the $g g$ initiated, FB-symmetric contribution has been included in the denominator as it should be. The asymmetry is sizable and may be used to obtain further constraints. As for the SM background to such an asymmetry, it is easy to see that the small parity violating EW contribution does not interfere with the strong amplitude, due to the different color structure and hence contribution to the FB asymmetry from the $Z$ exchange can be safely neglected. An additional contribution to the same also accrues [36, 37], the dominant part coming from the absorptive part of correction to the Born-level amplitude for $q \bar{q} \rightarrow t \bar{t}$. In fact, a similar contribution to the FB charge asymmetry exists even in the context of pure QED [38] in $e^{+} e^{-} \rightarrow \mu^{+} \mu^{-}$. The computations of the leading contributions to this asymmetry of Refs. [36, 37], find that an asymmetry as large as $4-5 \%$ is possible within the SM. This raises the issue of the separability of asymmetry caused by the axigluon contribution. Within the SM, the asymmetry arises from the contributions of a specific subclass of real and virtual radiative corrections and hence it has a particular phase space distribution. As Figures 5 and 9 of Refs. [36, 37] show, the asymmetry coming from the QCD source is larger at lower values of sub-process center-of-mass energy and at larger production angles of the top quark. On the other hand, the contribution to the FB asymmetry coming from a resonance will have a distinctive $m_{t \bar{t}}$ dependence and thus the two may be separated by making 
appropriate cuts on these two quantities. Such a study, though interesting, is beyond the scope of the present investigation. In view of the use of FB asymemtry in searching for unusual $t \bar{t}$ resonances, an analysis of this asymmetry taking into account higher order effects is certainly worth doing, but again quite beyond the scope of the current work.

\section{$5 t \bar{t}$ production at the LHC due to coloron/axigluons}

The situation of course is very different at the LHC as the $q \bar{q}$ fluxes are substantially smaller than the $g g$ fluxes and $t \bar{t}$ production is dominated by contribution from $g g$ initial state. However, this dominance is less severe as we go to larger $t \bar{t}$ invariant masses. Since, at the LHC, we would typically be interested in exploring larger axigluon (coloron) masses, it is wiser to concentrate on a data sample that has enhanced sensitivity to the mass range in question. Hence, in this case, instead of looking at the total integrated $t \bar{t}$ cross-sections, we consider the effect of the axigluon/coloron contribution on the $m_{t \bar{t}}$ spectrum. The large size of the top sample expected at the LHC ( 8 million pairs for $10 \mathrm{fb}^{-1}$ integrated luminosity), makes a study of such differential distribution meaningful. In Fig. 5, we show the $d \sigma / d m_{t \bar{t}}$ distribution as a function of $m_{t \bar{t}}$ at the LHC for both the cases, viz. the axigluon and the coloron. Let us reiterate that contributions from both, the $q \bar{q}$ and $g g$, initial states have been included in the numbers presented in Fig. 5. As can be seen from these plots, the resonances, though very broad,

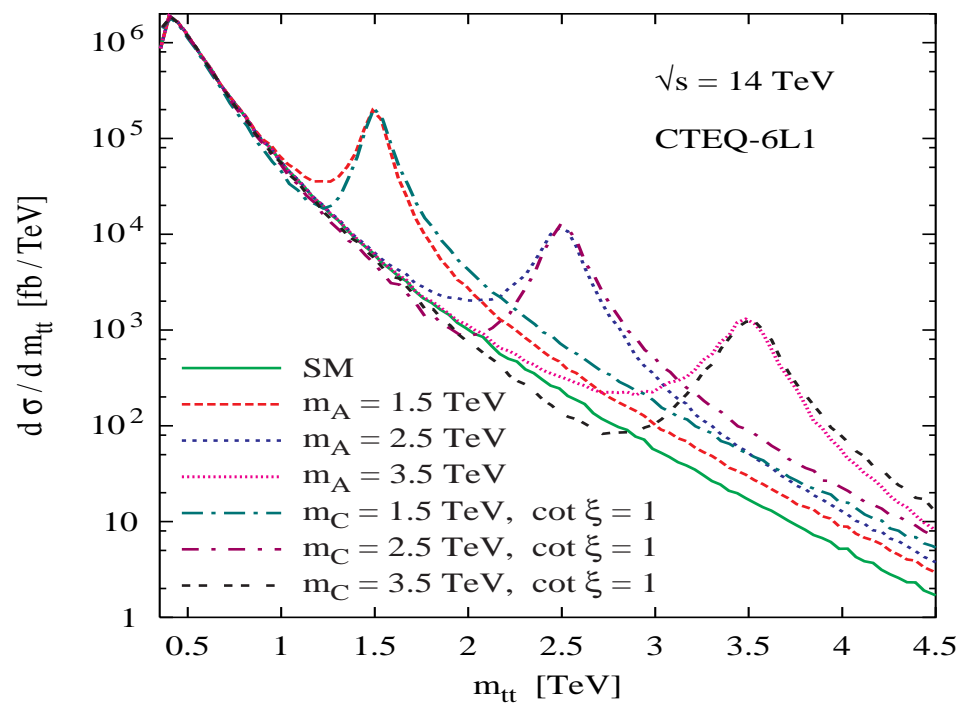

Figure 5: The expected $m_{t \bar{t}}$ spectrum at the LHC in presence of either axigluons or colorons of a specified mass. Also shown, for comparison, are the SM expectations.

will show up clearly over the continuum. Note, for example, for the first peak in Fig. 5, even assuming 
a $10 \%$ efficiency, there will be about $\sim 10^{4}$ events at $10 \mathrm{fb}^{-1}$. This should allow a cross-section measurement to a \% level. It may also be noticed that, precisely at the resonance, the cross-sections for the axigluon and the coloron for $\cot \xi=1$ for the same mass are indeed the same. (For, at that point, the additional cross section is well-approximated by $\sigma(p \bar{p} \rightarrow A / C) \times \operatorname{Br}(A / C \rightarrow t \bar{t})$, and for such large values of the boson masses, the branching fractions are nearly identical.) The distributions also show evidence of the aforementioned interference effects in case of the coloron. While this is destructive for $m_{t \bar{t}}<m_{C}$, it is the opposite for $m_{t \bar{t}}>m_{C}$. It can be easily seen that, for the same mass, the coloron cross-section should be asymptotically bigger than the axigluon cross-section by about a factor 2 as is seen in Fig. 5 .

The LHC being a $p p$ machine, the possibility of constructing a FB asymmetry does not exist. Since the axigluon has only a pure pseudo-vectorial coupling, a measurement of the net $t$ polarisation will also not probe the difference in the nature of the coupling between the axigluon and the coloron unlike the case of (say) an extra $Z^{\prime}$ present in many extensions of the Standard Model [15]. Indeed as Eqs.(18 \&4) show, the only dependence on polarizations is through the product of the two ( $t$ and $\bar{t})$ helicities and thus only a variable sensitive to this product can exploit this aspect. An example is afforded by

$$
\mathcal{R}_{\Delta}\left(m_{t t}\right) \equiv\left[\int_{m_{t t}-\Delta}^{m_{t t}+\Delta} d m_{t t} \frac{d \sigma_{-}}{d m_{t t}}\right]\left[\int_{m_{t t}-\Delta}^{m_{t t}+\Delta} d m_{t t} \frac{d \sigma_{+}}{d m_{t t}}\right]^{-1},
$$

where $\sigma_{ \pm}$refer to the cross sections for the product of the $t$ and $\bar{t}$ helicities to be \pm 1 respectively. Ideally, the interval $\Delta$ is to be chosen so as to maximize the sensitivity, and would nominally be a function of the width of the boson in question and the experimental accuracy in measuring $m_{t t}$. Rather than do this, we adopt two nominal values of $\Delta=0.1 m_{\text {Boson }}$ and $\Delta=0.2 m_{\text {Boson }}$ given the fact that the first choice well approximates $\Delta \simeq \Gamma_{\text {Boson }}$.

In Fig, 6 , we exhibit this ratio, evaluated at $m_{t t}=m_{A}\left(m_{C}\right)$ as a function of the boson mass. Clearly, in either case, the enhancement, with respect to the SM, of the $\lambda \bar{\lambda}=-1$ channel is much more marked than that for the $\lambda \bar{\lambda}=+1$ channel. Furthermore, this also differentiates between the axigluon and coloron modes for comparable values of couplings. This discriminator is in addition over the invariant mass distribution (as displayed in Fig (5). While it is true that an experimental measurement of this ratio (essentially a measurement of the correlation of the spin of the $t$ quark with the spin of the $\bar{t}$ ) would be a challenging one, it will not be impossible. In fact, possibilities of probing the heavy KK graviton resonances or the Heavy Higgs through such spin-spin correlations, have been studied in detail [39, 40] in the context of the ATLAS detector. This study shows that the asymmetry between like spin and unlike spin-pair can be measured with a precision of $4 \%$, with $10 \mathrm{fb}^{-1}$ luminosity. It is also worth pointing out that the dependence on the integral interval $\Delta$ while substantial, is not so large as to invalidate the efficacy of this observable. 

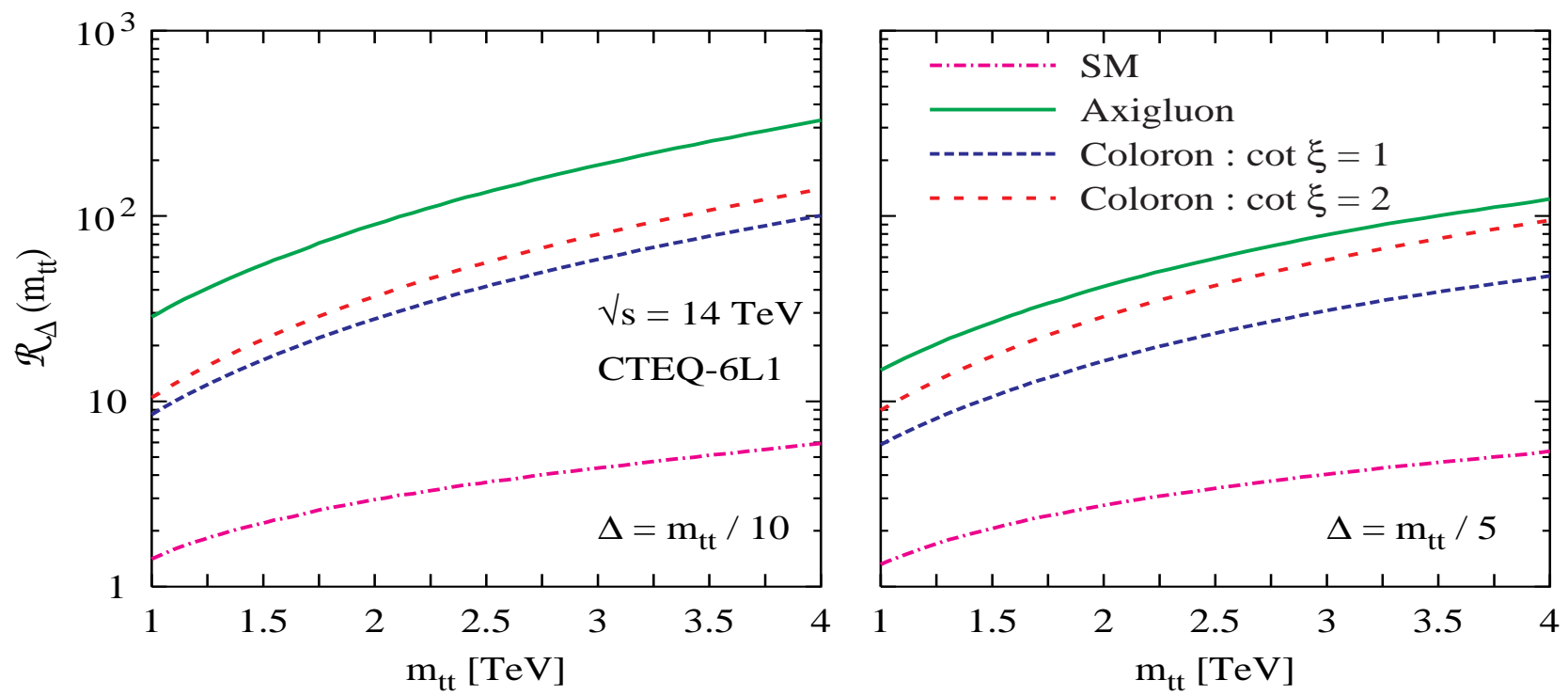

Figure 6: The ratio of the partial cross-sections $\mathcal{R}_{\Delta}\left(m_{t t}=m_{\text {Boson }}\right)$ - see Eqn. (8) - as a function of the boson mass. The two panels correspond to different values of $\Delta$.

\section{Summary}

We have investigated the contribution to the $t \bar{t}$ production at the Tevatron, in the presence of strongly interacting, non-standard spin-1 particles such as axigluons or flavour universal colorons. We find that the CDF Run-II data on $t \bar{t}$ production rule out axigluon masses $m_{A}$ up to $910(920) \mathrm{GeV}$ at $95 \%-(90 \%)$ confidence level. For the flavour universal colorons, the constraints depend on the mixing angle $\xi$ in a non monotonic way. For $\cot \xi=1$, the data rule out $m_{C} \lesssim 800(805) \mathrm{GeV}$ at $95 \%(90 \%)$ confidence level, as well as $895 \lesssim m_{C} \lesssim 1965\left(880 \lesssim m_{C} \lesssim 2475\right) \mathrm{GeV}$ at $95 \%(90 \%)$ C.L. On the other hand, for $\cot \xi=2$, the same data rule out $m_{C} \lesssim 955(960) \mathrm{GeV}$ and $1030 \lesssim m_{C} \lesssim 3200\left(1020 \lesssim m_{C} \lesssim 3250\right)$ $\mathrm{GeV}$ at $95 \%-(90 \%)$ C.L. We correct the formulae in literature for the axigluon mediated $t \bar{t}$ production cross-section as well as for the FB asymmetry expected at a $\bar{p} p$ collider in this case. We also point out that for the range of large axigluon/coloron masses, being probed at the Tevatron, the approximation of the incoherent sum of the resonance and the background, used normally in the analyses so far, will not be sufficient due to the large width of the boson. This fact, along with the differences in the $t \bar{t}$ decay width between a coloron and an axigluon, makes the expected cross-section for a flavour universal coloron for $\cot \xi=1$, different from that for an axigluon of the same mass, even for the dijet channel. We have further shown that the former also has the effect of making the contribution of the parity even interference term negative for the coloron case, causing the cross-section to dip below the SM value for certain values of $m_{C}$ depending on $\cot \xi$. We compute the FB asymmetry using the 
corrected formulae and show that it can be used to discriminate the axigluon contribution from the $\mathrm{SM}$ as well as from the coloron case. We further find that at the LHC, in spite of the dominance of the $g g$ initial state, the axigluon/coloron contribution can be seen clearly in the $m_{t \bar{t}}$ spectrum. We further suggest a variable constructed using top/anti-top polarizations which can discriminate the axigluon or coloron contribution from that of a gluon as well as from each other.

\section{Acknowledgments}

We wish to acknowledge discussions with Prof. S.D. Rindani. D.C. acknowledges support from the Department of Science and Technology, India under project number SR/S2/RFHEP-05/2006. R.M.G., R.K.S and K.W. wish to acknowledge support from Indo French Centre for Promotion of Advanced Research Project 3004-B.

\section{References}

[1] LEP Electroweak Working Group, http://lepewwg.web.cern.ch/LEPEWWG/

[2] J. Alcaraz et al. [LEP Collaboration], arXiv:hep-ex/0612034.

[3] For a review, see for example, R. M. Godbole, hep-ph/0205114, Part A, Volume 4, Jubilee Issue of the Indian Journal of Physics, pp. 44-83, 2004, Guest Editors: A. Raychaudhury and P. Mitra.

[4] C. T. Hill and E. H. Simmons, Phys. Rept. 381 (2003) 235-402, hep-ph/0203079.

[5] See for example discussions in, G. Weiglein et al. [LHC/LC Study Group], Phys. Rept. 426 (2006) 47 arXiv:hep-ph/0410364.

[6] C. T. Hill and S. J. Parke, Phys. Rev. D 49, 4454 (1994) arXiv:hep-ph/9312324.

[7] S. Cabrera [CDF and D0 Collaboration], FERMILAB-CONF-06-228-E, Jul 2006. 4pp. Presented at 14th International Workshop on Deep Inelastic Scattering (DIS 2006), Tsukuba, Japan, 20-24 Apr 2006.

[8] K. Lannon [CDF Collaboration], arXiv:hep-ex/0612009.

[9] M. Beneke et al., arXiv:hep-ph/0003033 and references therein.

[10] M. Guchait, F. Mahmoudi and K. Sridhar, arXiv:hep-ph/0703060.

[11] K. Agashe, A. Belyaev, T. Krupovnickas, G. Perez and J. Virzi, arXiv:hep-ph/0612015. 
[12] B. Lillie, L. Randall and L. T. Wang, arXiv:hep-ph/0701166.

[13] M. Arai, N. Okada, K. Smolek and V. Simak, Phys. Rev. D 70, 115015 (2004) arXiv:hep-ph/0409273; Phys. Rev. D 75 (2007) 095008 arXiv:hep-ph/0701155].

[14] R. M. Harris, C. T. Hill and S. J. Parke, arXiv:hep-ph/9911288.

[15] G. Azuelos, B. Brelier, D. Choudhury, P.-A. Delsart, R.M. Godbole, S.D. Rindani, R.K. Singh and K. Wagh, in arXiv:hep-ph/0602198; 197-205.

[16] P. H. Frampton and S. L. Glashow, Phys. Lett. B 190, 157 (1987).

[17] P. H. Frampton and S. L. Glashow, Phys. Rev. Lett. 58, 2168 (1987).

[18] R. S. Chivukula, A. G. Cohen and E. H. Simmons, Phys. Lett. B 380, 92 (1996) arXiv:hep-ph/9603311.

[19] J. Bagger, C. Schmidt and S. King, Phys. Rev. D 37, 1188 (1988).

[20] L. M. Sehgal and M. Wanninger, Phys. Lett. B 200 (1988) 211.

[21] F. Abe et al. [CDF Collaboration], Phys. Rev. D 55, 5263 (1997) arXiv:hep-ex/9702004.

[22] M. P. Giordani [CDF and D0 Collaborations], Eur. Phys. J. C 33, S785 (2004).

[23] M. A. Doncheski and R. W. Robinett, Phys. Rev. D 58, 097702 (1998) arXiv:hep-ph/9804226.

[24] For more details, see, W. M. Yao et al. [Particle Data Group], J. Phys. G 33 (2006) 1.

[25] M. A. Doncheski and R. W. Robinett, arXiv:hep-ph/9706490.

[26] C. T. Hill, Phys. Lett. B 266, 419 (1991).

[27] C. T. Hill, Phys. Lett. B 345 (1995) 483 arXiv:hep-ph/9411426.

[28] D. A. Dicus, B. Dutta and S. Nandi, Phys. Rev. D 51, 6085 (1995) arXiv:hep-ph/9412370].

[29] E. H. Simmons, Phys. Rev. D 55, 1678 (1997) arXiv:hep-ph/9608269.

[30] M. B. Popovic and E. H. Simmons, Phys. Rev. D 58, 095007 (1998) arXiv:hep-ph/9806287.

[31] R. S. Chivukula, B. A. Dobrescu and J. Terning, Phys. Lett. B 353, 289 (1995) arXiv:hep-ph/9503203.

[32] I. Bertram and E. H. Simmons, Phys. Lett. B 443 (1998) 347 arXiv:hep-ph/9809472.

[33] B. L. Combridge, Nucl. Phys. B 151 (1979) 429. 
[34] J. Pumplin, D. R. Stump, J. Huston, H. L. Lai, P. Nadolsky and W. K. Tung, JHEP 0207 (2002) 012 arXiv:hep-ph/0201195].

[35] J. M. Campbell, J. W. Huston and W. J. Stirling, Rept. Prog. Phys. 70 (2007) 89 arXiv:hep-ph/0611148.

[36] J. H. Kuhn and G. Rodrigo, Phys. Rev. Lett. 81, 49 (1998) arXiv:hep-ph/9802268.

[37] J. H. Kuhn and G. Rodrigo, Phys. Rev. D 59, 054017 (1999) arXiv:hep-ph/9807420.

[38] F. A. Berends, K. J. F. Gaemers and R. Gastmans, Nucl. Phys. B 63, 381 (1973).

[39] K. Smolek and V. Simak, Czech. J. Phys. 54 (2004) A451.

[40] F. Hubaut, E. Monnier, P. Pralavorio, K. Smolek and V. Simak, Eur. Phys. J. C 44S2 (2005) 13 arXiv:hep-ex/0508061. 